\title{
Low carrier concentration leads to high in-plane thermoelectric performance in $\mathbf{n}$-type SnS crystals
}

\author{
Wenke $\mathrm{He}^{1}$, Tao Hong ${ }^{1,2}$, Dongyang Wang ${ }^{1}$, Xiang $\mathrm{Gao}^{2}$ and Li-Dong Zhao ${ }^{1 *}$
}

\begin{abstract}
As a simple binary compound, p-type SnS shows great competitiveness in thermoelectrics due to the certain appealing carrier and phonon transport behaviors, coupled with its cost-effectiveness, earth-abundance and environmental compatibility. To promote the application of low-cost thermoelectric devices, we synthesized n-type SnS crystals through bromine doping. Herein, we report a high in-plane power factor of $\sim 28 \mu \mathrm{W} \mathrm{cm} \mathrm{cm}^{-1} \mathrm{~K}^{-2}$, and attribute it to an outstanding in-plane carrier mobility in the crystal form and the large Seebeck coefficient benefitting from the low carrier concentration. The calculations of elastic properties show that the low lattice thermal conductivity in SnS is closely related to its strong anharmonicity. Combining the excellent electrical transport properties with low thermal conductivity, a final $Z T$ of $\sim 0.4$ is attained at $300 \mathrm{~K}$, projecting a conversion efficiency of $\sim 5 \%$ at $873 \mathrm{~K}$ along the in-plane direction.
\end{abstract}

Keywords: tin sulfide, n-type, layered structure, carrier concentration, thermoelectric transports

\section{INTRODUCTION}

To cope with the issues of worldwide fossil fuel shortage and the serious environmental pollution caused by excessive consumption of this resource, many new energy technologies have emerged and developed over the past decades [1-3]. Among them, thermoelectric conversion is deemed to be a thriving energy utilization technology as it can generate electrical power through harvesting exhausted heat and realize solid-state cooling by applying electric current. The thermoelectric conversion efficiency for a given material is determined by a dimensionless figure of merit, defined as $Z T=S^{2} \sigma T / \kappa$, where $S, \sigma, T$ and $\kappa$ are the Seebeck coefficient, electrical conductivity, absolute temperature in Kelvin and total thermal conductivity (sum of the contributions from lattice thermal conductivity $\kappa_{\text {lat }}$ and electronic thermal conductivity $\kappa_{\text {ele }}$ ), respectively [4-6]. Under great explorations and efforts, increasing progress in advanced synthesis techniques [7-10], modern theories [11-13] on thermoelectric transports and strategies for improving thermoelectric performance have been made in previous decades. In addition, it is particularly important to explore and research new thermoelectric materials to meet the market demands of low cost, high effectiveness, component nontoxicity and environmental compatibility [14]. Tin sulfide ( $\mathrm{SnS}$ ) is one of the most representative thermoelectric candidates in recent years [15-17].

Unlike traditional extensively studied thermoelectrics (such as $\mathrm{Bi}_{2} \mathrm{Te}_{3}[8,18]$, PbTe [7,19,20], and GeTe [21,22]), $\mathrm{SnS}$ is made of low-cost or no-toxic elements, and crystallized in layered structure with lower symmetry and possesses large bandgap. This kind of material has the following characteristics. First, the carrier mobility can be achieved orders of magnitude improvement by utilizing its layered structure to grow crystals compared to the polycrystalline phase [16,23]. Although wide-bandgap semiconductors have intrinsically low carrier concentration, high carrier mobilities in crystals can compensate the deterioration in electrical conductivity $(\sigma=n e \mu)$. Further, through effective doping, the electrical conductivities in the form of crystals are superior to the polycrystalline $\mathrm{SnS}$, as well as the multi-band transport effects can be activated to enhance the Seebeck coefficient owing to its complex electronic band structure caused by the asymmetric crystal structure $[15,16]$. Consequently, the excellent electrical transport properties (power factor, $\mathrm{PF}=S^{2} \sigma$ ) in $\mathrm{SnS}$ crystals can be comparable to traditional high-performance thermoelectrics, and even beyond them at low temperatures especially [17]. Not only the electrical performance improvement benefits from these features, but also the thermal conductivity is related to them. Low symmetric structure means the complexity of

\footnotetext{
${ }^{1}$ School of Materials Science and Engineering, Beihang University, Beijing 100191, China

${ }^{2}$ Center for High Pressure Science and Technology Advanced Research (HPSTAR), Beijing 100094, China

* Corresponding author (email: zhaolidong@buaa.edu.cn)
} 
chemical bondings (e.g., anharmonic bondings) in materials, which bring about strong anharmonicity, thus leading to a low lattice thermal conductivity $\kappa_{\text {lat }}[24,25]$. The $\kappa_{\text {lat }}$ can reach $\sim 0.4 \mathrm{~W} \mathrm{~m}^{-1} \mathrm{~K}^{-1}$ in the high-temperature region in polycrystalline SnS [26,27], approaching the theoretical limit. Moreover, large bandgap can avoid the adverse effect on thermal conductivity of bipolar diffusion from intrinsic excitation at high temperatures, which usually occurs in semiconductors with narrow bandgaps. Thus, combining the electrical and thermal transports, these characteristics in SnS crystals can broaden the temperature range of high $Z T$ s rather than confine the maximum $Z T$ s within a narrow range $[14,17]$. Wide-ranged high ZTs (namely, high average ZT) are conducive to the overall thermoelectric conversion efficiency. Therefore, low-cost SnS becomes a competitive candidate for thermoelectric device applications.

Based on the above characteristics, increasing progress on the thermoelectric performance improvement has been made in p-type SnS recently. As is well known, a thermoelectric device consists of p-type and n-type legs. Therefore, it is of great importance to develop n-type SnS and match its p-type counterpart to promote the applications of this low-cost thermoelectric device with potential high economic benefits. To date, several attempts on how to realize $n$-type SnS have been conducted, including aliovalent $(\mathrm{Pb})$ or isovalent $(\mathrm{Sb}, \mathrm{Bi})$ ion doping in Sn sites to fabricate thin films [28-30], or halogen anions $(\mathrm{Cl}, \mathrm{Br})$ substitution in $\mathrm{S}$ sites to prepare bulk polycrystalline SnS [31,32]. The carrier concentration and carrier mobility, however, are too low to achieve good electrical conductivity for thermoelectrics. By the methods of crystal growth, the carrier mobility can achieve a huge improvement in SnS. Iguchi et al. [33] successfully fabricated n-type SnS single crystals using a self-flux method, the carrier mobility reaches $252 \mathrm{~cm}^{2} \mathrm{~V}^{-1} \mathrm{~s}^{-1}$ with a carrier concentration of $3 \times 10^{17} \mathrm{~cm}^{-3}$. Subsequently, Kawanishi et al. [34] reported a growth method of n-type SnS single crystals through halogen-doping from Snbased flux, with the carrier mobilities up to 155 and $154 \mathrm{~cm}^{2} \mathrm{~V}^{-1} \mathrm{~s}^{-1}$ as well as carrier concentrations up to 4.5 $\times 10^{17}$ and $7.6 \times 10^{17} \mathrm{~cm}^{-3}$ for $\mathrm{SnS}$ crystals with $\mathrm{Cl}$ and $\mathrm{Br}$ doping, respectively. Although these crystal-growth methods have improved the electrical conductivity of this material, they are limited by the thickness of layers, which is unfavorable for thermoelectric performance measurements and the device applications. Therefore, it is vital to grow large-sized n-type SnS bulk crystals. Recently, Yin et al. [35] reported a good-sized n-type $\mathrm{SnS}_{0.95}$ crystal using a temperature-gradient method, whose in-plane carrier mobility is $92 \mathrm{~cm}^{2} \mathrm{~V}^{-1} \mathrm{~s}^{-1}$ with carrier concentration of $1.7 \times 10^{18} \mathrm{~cm}^{-3}$. Hu et al. [32] prepared a n-type Br-doped SnS crystal, whose out-of-plane carrier mobility can reach $350 \mathrm{~cm}^{2} \mathrm{~V}^{-1} \mathrm{~s}^{-1}$. Generally, the in-plane carrier mobility in the SnS crystal is superior to the out-of-plane one, and the electrical conductivity will be better along the in-plane direction. Under these motivations mentioned above, we have grown a large-sized n-type Br-doped SnS crystal and investigated its in-plane thermoelectric transport properties in this work. The PF can attain to $\sim 28 \mu \mathrm{W} \mathrm{cm}{ }^{-1} \mathrm{~K}^{-2}$ at $300 \mathrm{~K}$, profiting from its high in-plane carrier mobility of $1268 \mathrm{~cm}^{2} \mathrm{~V}^{-1} \mathrm{~s}^{-1}$. Finally, a maximum $Z T$ of $\sim 0.4$ at $323 \mathrm{~K}$ is achieved, and the theoretical conversion efficiency reaches to $\sim 5 \%$ at $823 \mathrm{~K}$.

\section{RESULTS AND DISCUSSION}

$\mathrm{SnS}$ is a simple binary compound with two-dimensional (2D) structure, and it will undergo a displacive-type structure transition from Pnma to $\mathrm{Cmcm}$ structure at high temperatures $(873 \mathrm{~K})$ as the temperature rises [36]. Along the in-plane direction ( $b-c$ plane), the atoms are arranged in a periodic spring-like structure (Fig. 1a). In the $b-c$ plane, each atom is surrounded by four coordination atoms, forming a mesh-like structure (Fig. 1b). This 2D layered structure is conducive to carrier migration in the plane. Motivated by its high in-plane carrier mobility, we have grown SnS crystals with $\mathrm{Br}$ doping to realize the n-type conduction for the investigation on its in-plane thermoelectric transport properties (Fig. 1c).
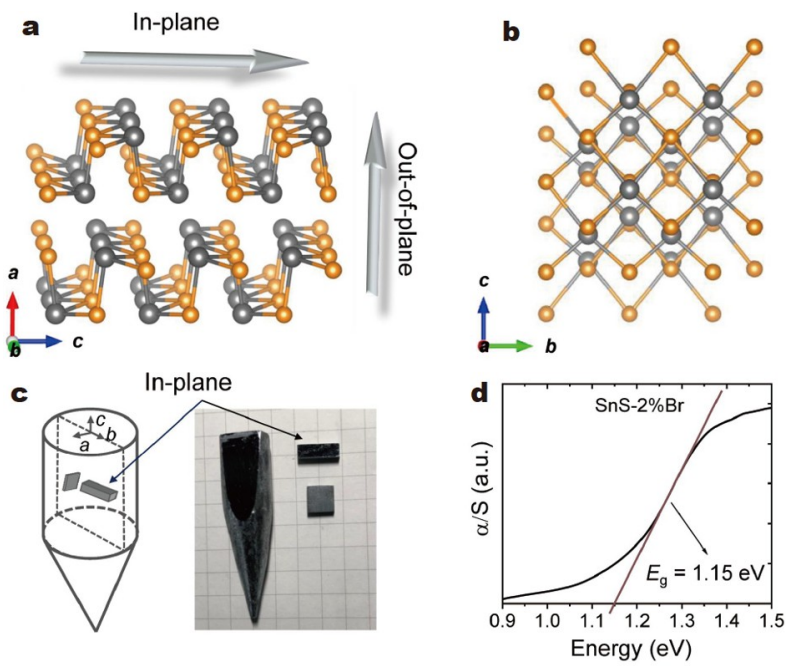

Figure 1 Crystal structure of SnS along the (a) $b$-c plane (in-plane) and (b) $a$-axis (out-of-plane). (c) A diagram shows how to cut samples along the in-plane direction, and the corresponding crystal cleaved along the (100) plane and sample for measurements. (d) Optical absorption spectrum and band gap of the Br-doped SnS. 
Also, the optical band gap $\left(E_{\mathrm{g}}\right)$ in Br-doped $\mathrm{SnS}$ is measured and a value of $1.15 \mathrm{eV}$ is obtained in Fig. 1d, which is consistent with the reported data in p-type $\operatorname{SnS}[16,26]$.

To verify the successful doping of $\mathrm{Br}$, we carried out scanning transmission electron microscopy (STEM) measurements. An area of the cleavage plane was selected to conduct the characterization on distribution of elements (Fig. 2a). The corresponding energy dispersive Xray spectroscopy (EDS) mapping images of $\mathrm{Br}, \mathrm{Sn}$ and $\mathrm{S}$ shown in Fig. 2b-d demonstrate uniform distributions of the elements. It indicates that $\mathrm{Br}$ atoms successfully substitute a fraction of $S$ sites, supporting the evidence of $\mathrm{n}$-type SnS from the view of microstructure.

In order to research the in-plane thermoelectric transports in n-type SnS, the thermoelectric property measurements of samples were performed. The measured Hall carrier concentration is $4.46 \times 10^{17} \mathrm{~cm}^{-3}$ at $300 \mathrm{~K}$, and obviously, this value does not reach the optimal carrier concentration as an ideal thermoelectric material. However, lower carrier concentration leads to higher carrier mobility as less ionized impurity scattering, coupled with higher carrier mobility in the form of crystals. These two factors bring about high in-plane carrier mobility of $1268 \mathrm{~cm}^{2} \mathrm{~V}^{-1} \mathrm{~s}^{-1}$ at $300 \mathrm{~K}$. Therefore, the in-plane electrical conductivity can attain to $92 \mathrm{~S} \mathrm{~cm}^{-1}$, much higher than the out-of-plane value in Br-doped SnS [32]
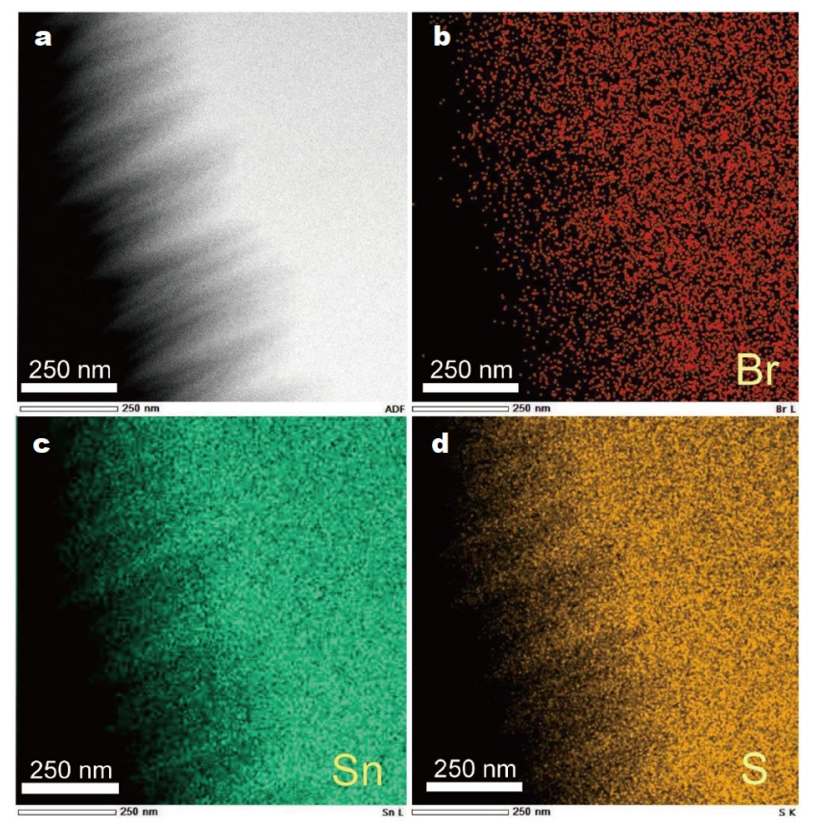

Figure 2 (a) Annular dark-field (ADF)-STEM image of an area on the cleavage plane. $(\mathrm{b}-\mathrm{d})$ The corresponding $\mathrm{Br}, \mathrm{Sn}$ and $\mathrm{S}$ distributions in the area shown in (a). The EDS mapping results show that the elements are distributed uniformly in Br-doped SnS.
(Fig. 3a). In addition, compared with the polycrystalline sample perpendicular to the spark plasma sintering (SPS) direction [32] (can be roughly considered as the $b-c$ plane direction in crystals), the in-plane electrical conductivity is far higher than the former (inset of Fig. 1a). Obviously, the electrical conductivity declines as the temperature rises, which is consistent with a temperature-dependent lattice scattering characteristic. This behavior is well illustrated by the temperature-dependent carrier mobility (Fig. 3b). The temperature-dependent carrier concentration is slightly changed over an entire temperature range (Fig. 3b), indicating a typical feature of doped semiconductors. Benefitting from the overall low carrier concentration, however, the Seebeck coefficient can reach as high as $-560 \mu \mathrm{V} \mathrm{K}^{-1}$ at $300 \mathrm{~K}$ and remains within a range from $\sim-500$ to $-600 \mu \mathrm{V} \mathrm{K} \mathrm{K}^{-1}$ with rising temperature (Fig. 3c). The reported Seebeck coefficients in the polycrystalline sample are significantly higher than that in crystals (below $\sim 600 \mathrm{~K}$ ), which may come from much lower carrier concentration and more scattering mechanisms in polycrystalline SnS. Further, based on the single parabolic band (SPB) mode, we plotted the Pisarenko line to estimate the effective mass with increased carrier concentration [37,38]. The simulated effective mass $m^{*}$ is $\sim 1.2 m_{\mathrm{e}}$, and the in-plane Seebeck coefficient values from this work and reported by Yin et al. [35] are falling roughly on this line, which implies a single band transport feature at low carrier concentration in n-type $\mathrm{SnS}$ (Fig. 3d). On the basis of the measured carrier mobility $\mu_{\mathrm{H}}$, we also fitted a relation curve of carrier mobility with elevated carrier concentration (inset of Fig. 3d). The in-plane carrier mobility $\left(\mu_{\mathrm{H}}=1268 \mathrm{~cm}^{2} \mathrm{~V}^{-1} \mathrm{~s}^{-1}\right)$ is higher than the out-of-plane value $\left(\mu_{\mathrm{H}}=350 \mathrm{~cm}^{2} \mathrm{~V}^{-1} \mathrm{~s}^{-1}\right)$, which is attributed to the selective strong inter-layer scattering in $\mathrm{SnS}$ crystals. The carrier mobility in $\mathrm{SnS}_{0.95}$ crystal is $92 \mathrm{~cm}^{2} \mathrm{~V}^{-1} \mathrm{~s}^{-1}$ with carrier concentration of $1.7 \times$ $10^{18} \mathrm{~cm}^{-3}$, far below the value of this work. The main cause may arise from the vast $\mathrm{S}$ vacancies in $\mathrm{SnS}_{0.95}$, leading to vacancy-induced strong point-defect scattering.

The high in-plane carrier mobility in SnS crystals and the large Seebeck coefficient benefitting from the low carrier concentration result in a high in-plane PF $\sim 28 \mu \mathrm{W} \mathrm{cm}{ }^{-1} \mathrm{~K}^{-2}$ at $300 \mathrm{~K}$ (Fig. 4a). This in-plane value is comparable to that in p-type SnS crystals after carrier concentration optimization $[15,16]$. The in-plane PFs within the entire working temperature precede the outof-plane in SnS crystals, and exceed the polycrystalline SnS (inset of Fig. 4a). The weighted mobility $\left(\mu_{\mathrm{w}}\right)$ is a functional parameter to evaluate the electrical transport 

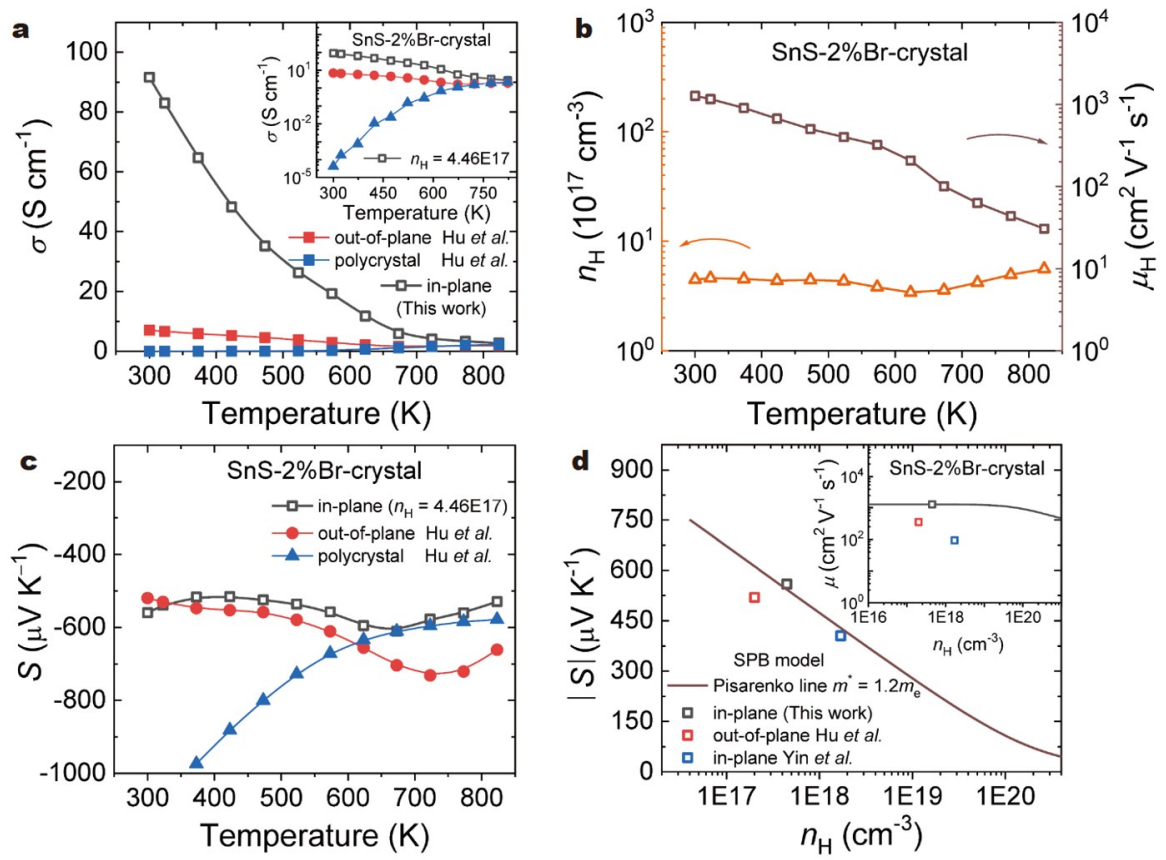

Figure 3 Electrical transport properties in Br-doped SnS crystals. (a) Electrical conductivity. (b) Carrier concentration and carrier mobility. (c) Seebeck coefficient. (d) Pisarenko line of the Seebeck coefficient as a function of carrier concentration at $300 \mathrm{~K}$. The inset presents the simulated line of carrier concentration-dependent carrier mobility. The reported data in n-type SnS crystals and polycrystalline SnS are also provided for comparison $[32,35]$.

behaviors as it can be derived from the experimental Seebeck coefficient and electrical conductivity [39-41]. Apparently, the in-plane weighted mobility in SnS crystals prevails over the out-of-plane as well as the polycrystalline form (Fig. 4b). Therefore, it is interesting to analyze the electronic band structure as it can provide a constructive guideline for the potential electrical performance optimization in n-type SnS. We have conducted the electronic band structure calculations through the density functional theory (DFT). It also demonstrates a complex conduction band structure is similar to the valance band structure in SnS (Fig. 4c). The first, second and third conduction band minimum (CBM1, CBM2 and CBM3) values lie along the $\Gamma-Y, \Gamma$ point and $\Gamma-Z$ direction, respectively. The energy difference between CBM1 and CBM2 is $\sim 0.2 \mathrm{eV}$, and the corresponding Fermi level of present carrier concentration does not reach the CBM2 or more band minimum apparently. Nevertheless, it indicates an underlying multi-band transport when the carrier concentration is optimized properly. To further inquire the orbital contributions of atoms in n-type SnS, the projected density of states (PDOS) near the band edges were calculated. The calculation results reveal that
Sn-p and S-p orbitals contribute equally to the total PDOS in valance band (p-type) while S-p dominates the PDOS in the conduction band (n-type) (inset of Fig. 4d). The specific contributions of atom along its three orbitals $\left(\mathrm{p}_{x}, \mathrm{p}_{y}\right.$ and $\left.\mathrm{p}_{z}\right)$ in SnS are presented in Fig. $4 \mathrm{~d}$, we can see that $\mathrm{S}-\mathrm{p}_{z}$ largely contributes to the total PDOS in valence band maximum (VBM), whereas $\mathrm{Sn}-\mathrm{p}_{y}$ primarily contributes to the total PDOS and then $S n-p_{z}$ takes the dominant status with increasing energy in the CBM. These contributions in conduction band manifest a tendency of the charge density distribution, which inclines to the in-plane direction at low concentration and then turns to the out-of-plane direction as the carrier concentration rises. These results support our experimental measurements that the in-plane electrical properties are superior to that of the out-of-plane at low concentrations, and imply an expectant out-of-plane electrical transport [42] once the carrier concentration is optimized in n-type SnS.

Owing to the layered structure and weak bonding in SnS, it shows strong anharmonicity, thus resulting in a low lattice thermal conductivity $[16,24,43]$. As is well known, the lattice thermal conductivity is strongly asso- 

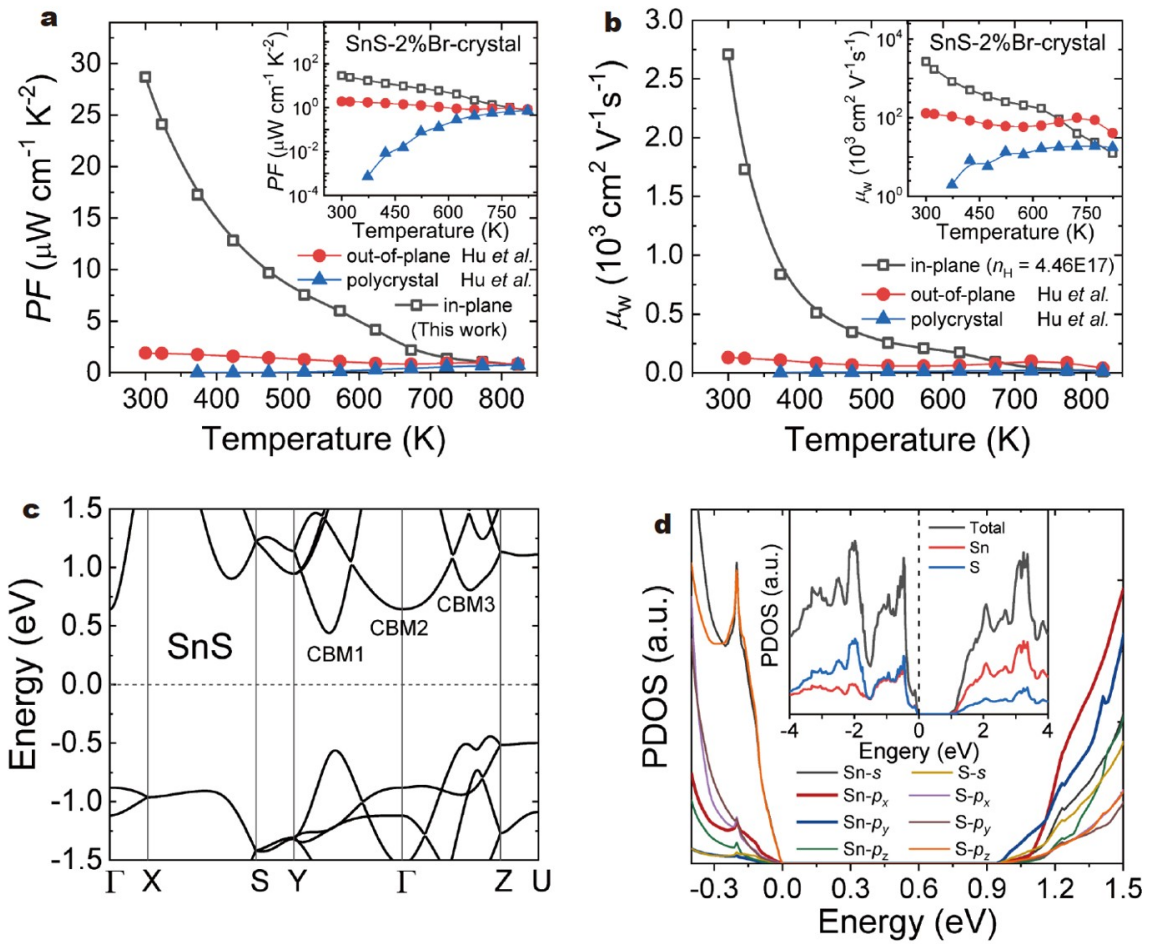

Figure 4 Electrical transport properties and electronic band structures in SnS crystals. (a) Power factor PF. (b) Weighted mobility. (c) Electronic band structure. (d) PDOS of SnS near the CBM and VBM. The inset shows the total DOS and the contribution of each atom.

ciated with the crystal structure and chemical bonding between atoms of materials, which can be reveled in elastic properties. To quantitatively describe the low thermal conductivity, the elastic property evaluations in $\mathrm{SnS}$ were conducted through ultrasonic pulse echo measurements. The elastic properties measured include average sound velocity $\left(v_{\mathrm{a}}\right)$, Young's modulus $(E)$, shear modulus $(G)$, Poisson ratio $\left(v_{\mathrm{p}}\right)$, Grüneisen parameter $(\gamma)$, and Debye temperature $\left(\theta_{\mathrm{D}}\right)$. The longitudinal $\left(v_{1}\right)$ and shear acoustic velocities $\left(v_{\mathrm{s}}\right)$ can be obtained using an ultrasonic instrument. On the basis of measured sound velocity, these physical parameters can be acquired as follows [44-46]:

$$
\begin{aligned}
& v_{\mathrm{a}}=\left[\frac{1}{3}\left(\frac{1}{v_{1}^{3}}+\frac{2}{v_{\mathrm{s}}^{2}}\right)\right]^{-1 / 3}, \\
& E=\frac{\rho v_{\mathrm{s}}^{2}\left(3 v_{1}^{2}-4 v_{\mathrm{s}}^{2}\right)}{\left(v_{1}^{2}-v_{\mathrm{s}}^{2}\right)}, \\
& v_{\mathrm{p}}=\frac{1-2\left(v_{\mathrm{s}} / v_{1}\right)^{2}}{2-2\left(v_{\mathrm{s}} / v_{1}\right)^{2}} \\
& G=\frac{E}{2\left(1+v_{\mathrm{p}}\right)}
\end{aligned}
$$

$$
\begin{aligned}
& \gamma=\frac{3}{2}\left(\frac{1+v_{\mathrm{p}}}{2-3 v_{\mathrm{p}}}\right), \\
& \theta_{\mathrm{D}}=\frac{h}{k_{\mathrm{B}}}\left[\frac{3 N}{4 \pi V}\right]^{1 / 3} v_{\mathrm{a}},
\end{aligned}
$$

where $\rho$ is the sample density, $h$ and $k_{\mathrm{B}}$ denote the Planck's constant and Boltzmann constant, respectively. $N$ is the number of atoms, and $V$ is the volume in a unit cell. The calculated physical parameters are shown in Table 1, and the elastic properties of other materials are provided for comparison $[43,47]$.

It is obvious that $\mathrm{SnS}$ and SnSe with layered structure possess larger Grüneisen parameters (2.28) than those of IV-VI group thermoelectric materials ( $\mathrm{SnTe}, \mathrm{PbTe}, \mathrm{PbSe}$, and $\mathrm{PbS}$ ) with cubic structure ( $\gamma$ within 1.65-1.75), which can well support the strong anharmonicity in layered materials like SnS and SnSe. Therefore, the layered structure and anisotropic chemical bonding in $\mathrm{SnS}$ are the main origins for its strong anharmonicity, thus resulting in low lattice thermal conductivity.

Further, we carried out the thermal conductivity measurements in SnS crystals. The total thermal conductivity in the plane is $2.32 \mathrm{~W} \mathrm{~m}^{-1} \mathrm{~K}^{-1}$ at room temperature and 
Table 1 The calculated elastic properties of SnS and other IV-VI group thermoelectric materials

\begin{tabular}{|c|c|c|c|c|c|c|}
\hline Parameters & SnS & SnSe & $\mathrm{SnTe}$ & $\mathrm{PbTe}$ & $\mathrm{PbSe}$ & $\mathrm{PbS}$ \\
\hline$v_{1}\left(\mathrm{~m} \mathrm{~s}^{-1}\right)$ & 3715 & 2730 & 3250 & 2910 & 3200 & 3450 \\
\hline$v_{\mathrm{s}}\left(\mathrm{m} \mathrm{s}^{-1}\right)$ & 1701 & 1250 & 1750 & 1610 & 1750 & 1900 \\
\hline$v_{\mathrm{a}}\left(\mathrm{m} \mathrm{s}^{-1}\right)$ & 1917 & 1409 & 1954 & 1794 & 1951 & 2118 \\
\hline$E(\mathrm{GPa})$ & 39.6 & 26.4 & 51.2 & 54.1 & 65.2 & 70.4 \\
\hline$G(\mathrm{GPa})$ & 14.5 & 9.7 & 19.7 & 21.1 & 25.3 & 27.4 \\
\hline$v_{\mathrm{p}}$ & 0.37 & 0.37 & 0.30 & 0.28 & 0.29 & 0.28 \\
\hline$\gamma$ & 2.28 & 2.28 & 1.75 & 1.65 & 1.69 & 1.67 \\
\hline$\theta_{\mathrm{D}}$ & 198 & 141 & 184 & 165 & 190 & 212 \\
\hline
\end{tabular}
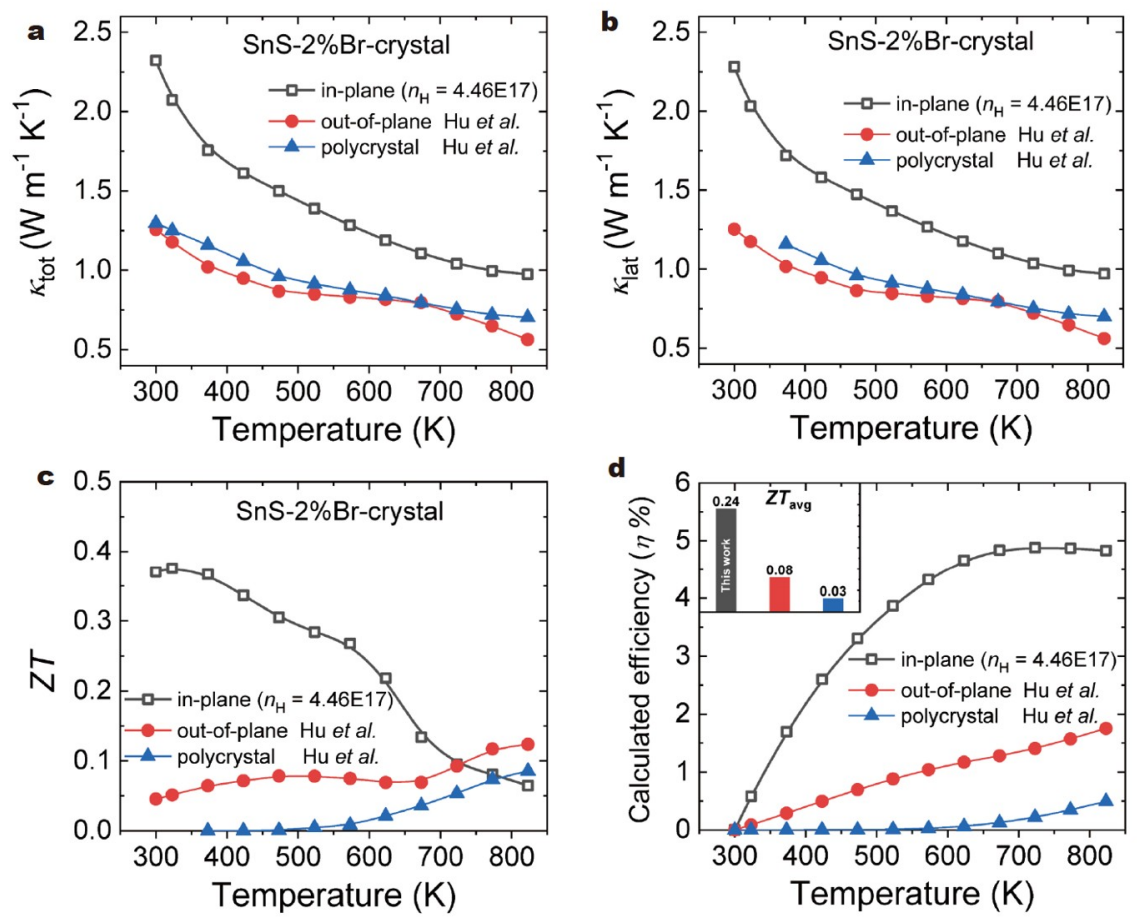

Figure 5 Thermal transport properties, $Z T$ values and efficiencies. (a) Total thermal conductivity. (b) Lattice thermal conductivity. (c) $Z T$ values. (d) Calculated conversion efficiency. The average $Z T$ values of samples are shown in the inset. The reported values of n-type SnS crystals and polycrystalline $\mathrm{SnS}$ are plotted for comparison [32].

decreases as the temperature rises (Fig. 5a). The in-plane thermal conductivities over the entire working temperature range are apparently higher than that of the out-ofplane in crystals. It should be noted that the low out-ofplane thermal conductivity in SnS mainly comes from the inter-layer phonon impediment, thus leading to strong phonon scattering. Similarly, the thermal conductivities are very low in polycrystalline samples compared with those of crystals along the in-plane direction, which originates from the strong grain boundary scattering. Due to the comparatively low carrier concentration in Br-doped $\mathrm{SnS}$, the contribution of thermal conductivity from the electron is small to the total thermal conductivity, which means that the thermal conductivity is dominated by the phonon transport from the lattice vibration (Fig. 5b). Combining the excellent PF with low $\kappa$, a maximum inplane $Z T$ of $\sim 0.4$ at $323 \mathrm{~K}$ is achieved in Br-doped SnS crystals. In addition, the in-plane $Z T$ values (before $\sim 700 \mathrm{~K}$ ) are obviously superior to the out-of-plane values in $\mathrm{SnS}$ crystals and the values in the polycrystalline form (Fig. 5 c). The average $Z T\left(Z T_{\text {avg }}\right)$ value at 300 to $823 \mathrm{~K}$ is calculated through Equation (7) $[37,38]$, and a $Z T_{\text {avg }}$ of $\sim 0.24$ is obtained along the in-plane direction in crystals (inset of Fig. 5d). This value is three times that of the out- 
of-plane value ( $\left.Z T_{\text {avg }} \sim 0.08\right)$, and eight times that of the polycrystalline $\mathrm{SnS}\left(Z T_{\text {avg }} \sim 0.03\right)$ [32]. Further, we calculated the thermoelectric conversion efficiency $(\eta)$ from the $Z T_{\text {avg }}$ by Equation (8) [48].

$Z T_{\text {avg }}=\frac{1}{T_{\mathrm{h}}-T_{\mathrm{c}}} \int_{T_{\mathrm{c}}}^{T_{\mathrm{h}}} Z T \mathrm{~d} T$,

$\eta=\frac{T_{\mathrm{h}}-T_{\mathrm{c}}}{T_{\mathrm{h}}} \frac{\sqrt{1+Z T_{\mathrm{avg}}}-1}{\sqrt{1+Z T_{\mathrm{avg}}}+T_{\mathrm{c}} / T_{\mathrm{h}}}$,

where $T_{\mathrm{c}}$ and $T_{\mathrm{h}}$ represent the cold and hot side temperature, respectively (here $T_{\mathrm{c}}$ is fixed at $300 \mathrm{~K}$ ). The final theoretical conversion efficiency in the plane can reach $\sim 5 \%$ at $873 \mathrm{~K}$ in SnS crystals, higher than the out-of-plane value of $\sim 2 \%$ and $\sim 0.5 \%$ in the polycrystalline sample (Fig. 5d).

\section{CONCLUSIONS}

In this work, $\mathrm{Br}$ doping realizes the n-type conduction behavior in SnS crystals, and the carrier concentration reaches $4.46 \times 10^{17} \mathrm{~cm}^{-3}$. A high in-plane $\mathrm{PF}$ of $28 \mu \mathrm{W} \mathrm{cm}{ }^{-1} \mathrm{~K}^{-2}$ is achieved at $300 \mathrm{~K}$ in SnS crystals, which is ascribed to the high in-plane carrier mobility in the crystal form and the large Seebeck coefficient benefitting from the low carrier concentration. The synergic effects of the excellent electrical properties and low lattice thermal conductivity bring about a peak $Z T$ of $\sim 0.4$ at room temperature, and the final conversion efficiency of $\sim 5 \%$ at $873 \mathrm{~K}$ is achieved along the in-plane direction. These results demonstrate that $\mathrm{n}$-type $\mathrm{SnS}$ is a competitive thermoelectric candidate for promoting the applications of this low-cost thermoelectric device with potential high economic benefits.

\section{Received 23 March 2021; accepted 9 April 2021; published online 16 June 2021}

1 Zhang X, Zhao LD. Thermoelectric materials: Energy conversion between heat and electricity. J Materiomics, 2015, 1: 92-105

2 Tan G, Zhao LD, Kanatzidis MG. Rationally designing high-performance bulk thermoelectric materials. Chem Rev, 2016, 116: 12123-12149

3 Shi XL, Zou J, Chen ZG. Advanced thermoelectric design: From materials and structures to devices. Chem Rev, 2020, 120: 73997515

4 Qin BC, Xiao Y, Zhou YM, et al. Thermoelectric transport properties of Pb-Sn-Te-Se system. Rare Met, 2017, 37: 343-350

5 Qu WW, Zhang XX, Yuan BF, et al. Homologous layered $\mathrm{InFeO}_{3}-$ $(\mathrm{ZnO})_{m}$ : New promising abradable seal coating materials. Rare Met, 2018, 37: 79-94

6 Wu D, Pei Y, Wang Z, et al. Significantly enhanced thermoelectric performance in $\mathrm{n}$-type heterogeneous BiAgSeS composites. Adv Funct Mater, 2014, 24: 7763-7771
7 Hsu KF, Loo S, Guo F, et al. Cubic $\mathrm{AgPb}_{m} \mathrm{SbTe}_{2+m}$ : Bulk thermoelectric materials with high figure of merit. Science, 2004, 303: 818-821

8 Poudel B, Hao Q, Ma Y, et al. High-thermoelectric performance of nanostructured bismuth antimony telluride bulk alloys. Science, 2008, 320: 634-638

9 Zhao LD, Lo SH, Zhang Y, et al. Ultralow thermal conductivity and high thermoelectric figure of merit in SnSe crystals. Nature, 2014, 508: 373-377

10 Pei Y, Chang C, Wang Z, et al. Multiple converged conduction bands in $\mathrm{K}_{2} \mathrm{Bi}_{8} \mathrm{Se}_{13}$ : A promising thermoelectric material with extremely low thermal conductivity. J Am Chem Soc, 2016, 138: 16364-16371

11 Pei Y, Gibbs ZM, Gloskovskii A, et al. Optimum carrier concentration in n-type PbTe thermoelectrics. Adv Energy Mater, 2014, 4: 1400486

12 Biswas K, He J, Blum ID, et al. High-performance bulk thermoelectrics with all-scale hierarchical architectures. Nature, 2012, 489: 414-418

13 Zhou Y, Zhao LD. Promising thermoelectric bulk materials with 2D structures. Adv Mater, 2017, 29: 1702676

14 Xiao Y, Zhao LD. Seeking new, highly effective thermoelectrics. Science, 2020, 367: 1196-1197

15 Wu H, Lu X, Wang G, et al. Sodium-doped tin sulfide single crystal: A nontoxic earth-abundant material with high thermoelectric performance. Adv Energy Mater, 2018, 8: 1800087

16 He W, Wang D, Dong JF, et al. Remarkable electron and phonon band structures lead to a high thermoelectric performance $Z T>1$ in earth-abundant and eco-friendly SnS crystals. J Mater Chem A, 2018, 6: 10048-10056

17 He W, Wang $\mathrm{D}, \mathrm{Wu} \mathrm{H}$, et al. High thermoelectric performance in low-cost $\mathrm{SnS}_{0.91} \mathrm{Se}_{0.09}$ crystals. Science, 2019, 365: 1418-1424

18 Pan Y, Li JF. Thermoelectric performance enhancement in n-type $\mathrm{Bi}_{2}(\mathrm{TeSe})_{3}$ alloys owing to nanoscale inhomogeneity combined with a spark plasma-textured microstructure. NPG Asia Mater, 2016, 8: e275

19 Xiao Y, Zhao LD. Charge and phonon transport in PbTe-based thermoelectric materials. npj Quant Mater, 2018, 3: 55

20 Xiao Y, Wu H, Cui J, et al. Realizing high performance n-type $\mathrm{PbTe}$ by synergistically optimizing effective mass and carrier mobility and suppressing bipolar thermal conductivity. Energy Environ Sci, 2018, 11: 2486-2495

21 Hong M, Zou J, Chen ZG. Thermoelectric GeTe with diverse degrees of freedom having secured superhigh performance. Adv Mater, 2019, 31: 1807071

22 Wu D, Zhao LD, Hao S, et al. Origin of the high performance in GeTe-based thermoelectric materials upon $\mathrm{Bi}_{2} \mathrm{Te}_{3}$ doping. J Am Chem Soc, 2014, 136: 11412-11419

23 Zhao LD, Chang C, Tan G, et al. SnSe: A remarkable new thermoelectric material. Energy Environ Sci, 2016, 9: 3044-3060

24 Chang C, Zhao LD. Anharmoncity and low thermal conductivity in thermoelectrics. Mater Today Phys, 2018, 4: 50-57

25 Wang D, Huang Z, Zhang Y, et al. Extremely low thermal conductivity from bismuth selenohalides with 1D soft crystal structure. Sci China Mater, 2020, 63: 1759-1768

26 Tan Q, Zhao LD, Li JF, et al. Thermoelectrics with earth abundant elements: Low thermal conductivity and high thermopower in doped SnS. J Mater Chem A, 2014, 2: 17302-17306

27 Zhou B, Li S, Li W, et al. Thermoelectric properties of SnS with Na-doping. ACS Appl Mater Interfaces, 2017, 9: 34033-34041 
28 Ran FY, Xiao Z, Toda Y, et al. n-Type conversion of SnS by isovalent ion substitution: Geometrical doping as a new doping route. Sci Rep, 2015, 5: 10428

29 Sinsermsuksakul P, Chakraborty R, Kim SB, et al. Antimonydoped tin(II) sulfide thin films. Chem Mater, 2012, 24: 4556-4562

30 Dussan A, Mesa F, Gordillo G. Effect of substitution of Sn for Bi on structural and electrical transport properties of SnS thin films. J Mater Sci, 2010, 45: 2403-2407

31 Huang ZC, Yao Y, Pei J, et al. Preparation and thermoelectric property of n-type SnS. J InOrg Mater, 2019, 34: 321-327

$32 \mathrm{Hu} \mathrm{X}, \mathrm{He} \mathrm{W}$, Wang D, et al. Thermoelectric transport properties of n-type tin sulfide. Scripta Mater, 2019, 170: 99-105

33 Iguchi $\mathrm{Y}$, Inoue K, Sugiyama $\mathrm{T}$, et al. Single-crystal growth of $\mathrm{Cl}-$ doped n-type SnS using $\mathrm{SnCl}_{2}$ self-flux. Inorg Chem, 2018, 57: 6769-6772

34 Kawanishi S, Suzuki I, Ohsawa T, et al. Growth of large single crystals of n-type SnS from halogen-added Sn flux. Cryst Growth Des, 2020, 20: 5931-5939

35 Yin Y, Cai J, Wang H, et al. Single-crystal growth of n-type $\mathrm{SnS}_{0.95}$ by the temperature-gradient technique. Vacuum, 2020, 182: 109789

36 Chattopadhyay T, Pannetier J, Von Schnering HG. Neutron diffraction study of the structural phase transition in SnS and SnSe. J Phys Chem Solids, 1986, 47: 879-885

37 Zhao LD, Tan G, Hao S, et al. Ultrahigh power factor and thermoelectric performance in hole-doped single-crystal SnSe. Science, 2016, 351: 141-144

38 Qin B, Wang D, He W, et al. Realizing high thermoelectric performance in p-type SnSe through crystal structure modification. J Am Chem Soc, 2018, 141: 1141-1149

39 Snyder GJ, Snyder AH, Wood M, et al. Weighted mobility. Adv Mater, 2020, 32: 2001537

40 Qin B, He W, Zhao LD. Estimation of the potential performance in p-type SnSe crystals through evaluating weighted mobility and effective mass. J Materiomics, 2020, 6: 671-676

$41 \mathrm{He}$, Qin B, Zhao LD. Predicting the potential performance in ptype SnS crystals via utilizing the weighted mobility and quality factor. Chin Phys Lett, 2020, 37: 087104

42 Chang $\mathrm{C}, \mathrm{Wu} \mathrm{M}, \mathrm{He} \mathrm{D}$, et al. 3D charge and 2D phonon transports leading to high out-of-plane $Z T$ in n-type SnSe crystals. Science, 2018, 360: 778-783

43 Xiao Y, Chang C, Pei Y, et al. Origin of low thermal conductivity in SnSe. Phys Rev B, 2016, 94: 125203

44 Wan C, Qu Z, He Y, et al. Ultralow thermal conductivity in highly anion-defective aluminates. Phys Rev Lett, 2008, 101: 085901

45 Kurosaki $\mathrm{K}$, Kosuga $\mathrm{A}$, Muta $\mathrm{H}$, et al. $\mathrm{Ag}_{9} \mathrm{TlTe}_{5}$ : A high-performance thermoelectric bulk material with extremely low thermal conductivity. Appl Phys Lett, 2005, 87: 061919

46 Cho JY, Shi X, Salvador JR, et al. Thermoelectric properties and investigations of low thermal conductivity in Ga-doped $\mathrm{Cu}_{2} \mathrm{GeSe}_{3}$. Phys Rev B, 2011, 84: 085207

47 Pei $\mathrm{Y}$, Zheng $\mathrm{L}, \mathrm{Li} \mathrm{W}$, et al. Interstitial point defect scattering contributing to high thermoelectric performance in SnTe. Adv Electron Mater, 2016, 2: 1600019

48 Zhang $\mathrm{X}$, Wang $\mathrm{D}, \mathrm{Wu} \mathrm{H}$, et al. Simultaneously enhancing the power factor and reducing the thermal conductivity of SnTe via introducing its analogues. Energy Environ Sci, 2017, 10: 2420-2431

Acknowledgements This work was supported by Beijing Natural Science Foundation (JQ18004), the National Key Research and Devel- opment Program of China (2018YFA0702100 and 2018YFB0703600), the National Natural Science Foundation of China (51772012), Shenzhen Peacock Plan team (KQTD2016022619565991), the National Postdoctoral Program for Innovative Talents (BX20200028), and the 111 Project (B17002). Zhao LD thanks the support from the National Science Fund for Distinguished Young Scholars (51925101).

Author contributions $\mathrm{He} \mathrm{W}$ and Zhao LD designed and synthesized the samples; Wang D carried out the DFT calculations; Hong T and Gao $\mathrm{X}$ performed STEM measurements; He W wrote the paper with support from Zhao LD. All authors contributed to the general discussion.

Conflict of interest The authors declare that they have no conflict of interest.

Supplementary information Experimental details are available in the online version of the paper.

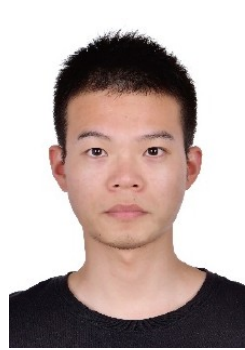

Wenke $\mathrm{He}$ is a $\mathrm{PhD}$ student in the School of Materials Science and Engineering at Beihang University, China. He received his BE degree from China Three Gorges University in 2016. He started his doctoral research as a member of LiDong Zhao's group in 2016. His main research interests focus on the crystal growth and performance optimization in thermoelectric materials with layered structures.

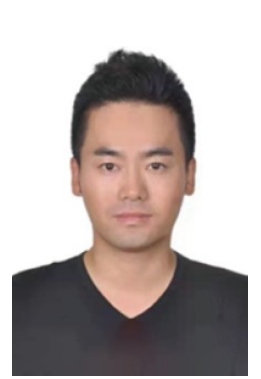

Li-Dong Zhao is a full professor of the School of Materials Science and Engineering at Beihang University, China. He received his $\mathrm{PhD}$ degree from the University of Science and Technology Beijing, China in 2009. He was a postdoctoral research associate at the Université Paris-Sud and Northwestern University from 2009 to 2014. His research interests include electrical and thermal transport behaviors in the compounds with layered structures. Group website: http://shi.buaa.edu.cn/zhaolidong/zh_CN/.

\section{在低载流子浓度的 $\mathrm{n}$ 型层状 $\mathrm{SnS}$ 晶体中实现高的面 内热电性能}

何文科 ${ }^{1}$, 洪涛 ${ }^{1,2}$, 王东洋 ${ }^{1}$, 高翔 ${ }^{2}$, 赵立东 ${ }^{1 *}$

摘要 硫化锡 $(\mathrm{SnS})$ 作为一种简单的二元化合物, 具有优异的电子 和声子输运特性, 加上其成本低、地球储量丰富、环境友好等优 点, 在热电材料领域显示出巨大的竞争力. 为了促进低成本热电器 件的发展和应用, 我们采用溴 $(\mathrm{Br})$ 掺杂生长 $\mathrm{SnS}$ 晶体实现其从本征 $\mathrm{p}$ 型向 $\mathrm{n}$ 型的转变. 此外, 得益于低的载流子浓度, 我们报道了 $\mathrm{n}$ 型 $\mathrm{SnS}$ 优异的面内载流子迁移率和较大的塞贝克系数, 从而导致一个高 的功率因子 $\mathrm{PF} \sim 28 \mu \mathrm{W} \mathrm{cm}{ }^{-1} \mathrm{~K}^{-2}$. 弹性性能计算表明, $\mathrm{SnS}$ 中的低晶 格热导率与其强非简谐性密切相关. 结合其优良的热电输运性能, 在室温下 $Z T$ 值达到了 0.4, $823 \mathrm{~K}$ 时其理论热电转化效率达到 $5 \%$. 